\title{
Interactive 3D Rapid-Prototyping Models
}

\author{
Carlo H. Séquin* \\ U.C. Berkeley, EECS, CS Division
}

\section{Introduction}

Today CAD programs are not just producing mind-blowing graphics pictures or convincing virtual models that can be interactively manipulated and changed. They also can yield tangible, physical artifacts, thanks to the development of many layered manufacturing techniques and the emergence of services such as Shapeways [2011] and Ponoko [2011], Creating, manipulating, and studying such $3 \mathrm{D}$ artifacts adds a whole new loop of feedback to the design process. This extra experience is also highly useful in the teaching of computer-aided design. In a graduate course on Solid Modeling and Rapid Prototyping we have used the design and realization of dissection puzzles as a training tool. Dissection puzzles are particularly valuable design objects, since they not only train spatial understanding, but they also drive home issues concerning accuracy and tolerances in a very "hands-on" manner. In this poster and in the accompanying models we demonstrate some of the dissection models that we studied and elucidate the modeling issues associated with each type.

\section{Dissection Geometries}

Figure 1 shows some of the Platonic solids dissected into congruent parts. These simple shapes are used to introduce the students to the concept of dissections.

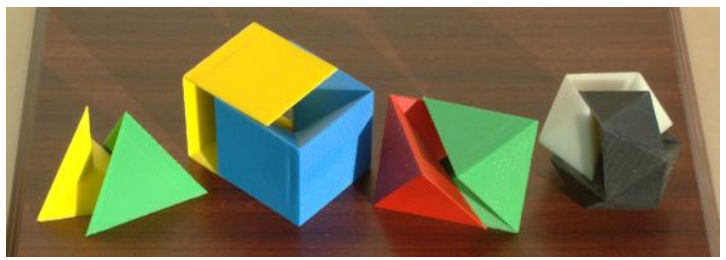

Figure 1: Hamiltonian dissections of Platonic solids.

The students were then given the task to devise dissections in which two or three congruent parts would separate with a helical twisting motion. Three student designs are displayed in Figure 2.
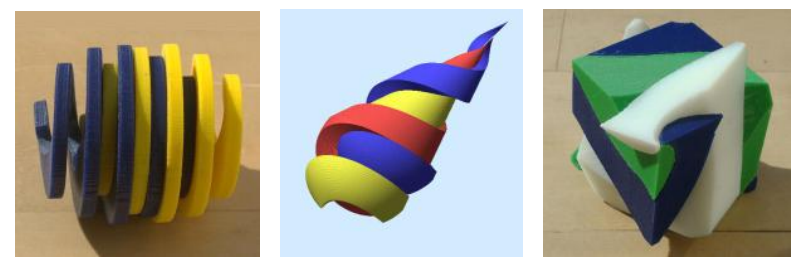

Figure 2: Simple helicoidal dissections.

A generalized method is then developed to create such puzzles. It starts with a sectioning in which the parts separate with a straight, linear motion along a shared axis (Fig.3a). All geometries are subsequently twisted in a helicoidal manner around this common axis (Fig.3b-d).
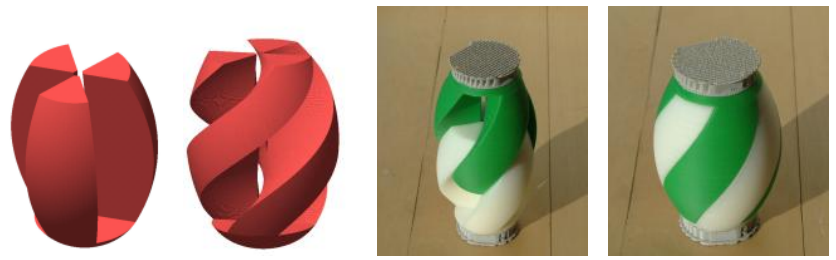

Figure 3: Multi-prong helicoidal partitioning.
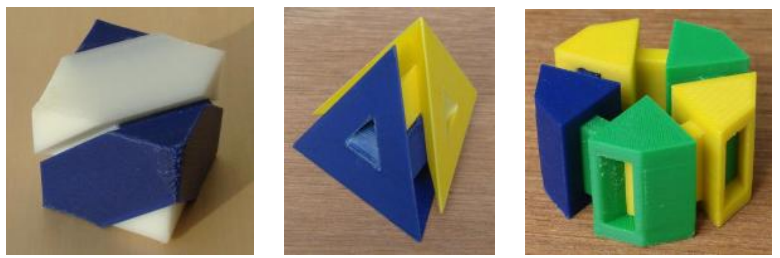

Figure 4: Dissection parts with genus greater than zero.

Some of the prongs belonging to one part may completely penetrate the other part via a through-hole. This permits the design of intriguing puzzles, where disconnected patches of one color appear in various locations on the puzzle surface, but still all belong to one and the same part ; thus each part has the topology of a donut with one or more holes (Fig.4a,b); It is quite challenging - and thus a good class exercise - to start from some pre-defined coloring pattern on a simple polyhedron and then trying to find a corresponding sectioning of the complete solid. It may even lead to "puzzles that cannot be taken apart with two hands" [Snoeyink 1993], but which need some coordinated action of all the parts for separation (Fig.4c).

\section{Addressing Fabrication Costs}

An intriguing little cubic burr puzzle, which was purchased from Shapeways for less than $10 \$$, was used to address issues of manufacturing costs. The original puzzle is very small - only $16 \mathrm{~mm}$ on a side (Fig. 5 top). We would like to have this puzzle at a scale at least 8 times larger. However, the price of such parts scales roughly with their volume, i.e., the third power of the scale factor. We looked at ways to redesign this puzzle, so that it would use substantially less building material. The winning solution employed an airy edge-frame for each unit-cubelet. This also has the advantage that the puzzle becomes rather transparent, so that one can study the inner workings of this puzzle. Moreover, the individual pieces would make nice constructivist sculptures when scaled up further.
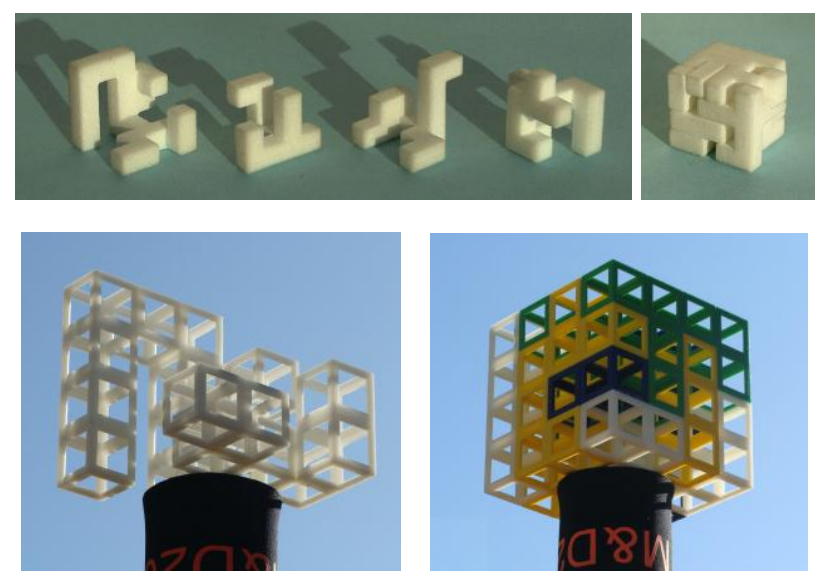

Figure 5: Top: Original cubic burr puzzle. Bottom: Enlarged, "transparent" re-design: Largest piece, and assembled puzzle.

\section{References}

Pocono 2011. http://www.ponoko.com/

ShaPEWAYs 2011. http://www.shapeways.com/

SNoeyink, J. and Stolfy, J. 1993. Objects that cannot be taken apart with two hands. SCG '93 San Diego, pp 247-256.

\footnotetext{
*e-mail: sequin@cs.berkeley.edu
} 